\title{
DICTONS ROMANS AVEC LES DOUZE MOIS : LA CARACTÉRISATION PARÉMIQUE ET MENSUELLE DE L'ANNÉE ${ }^{1}$
}

\author{
MARIA-REINA BASTARDAS RUFAT ${ }^{2}$, JOAN FONTANA I TOUS ${ }^{3}$, \\ JOSÉ ENRIQUE GARGALLO GIL ${ }^{4}$
}

\begin{abstract}
Romance Proverbs with the Twelve Months: the Paremical and Monthly Characterization of the Year. There are various types of weather and calendar proverbs in the Romance languages. Not only concerning their motivation, but also concerning their length. Among the proverbs referring to the months, some are very short, just two or three words, the so-called "minimal proverbs", while others are quite long, and curiously defy any kind of mnemonics. Our corpus will be made up of the latter type: thirteen proverbs mentioning all the twelve months of the year, which represent five different Romance varieties; from west to east: Portuguese (1 proverb), Spanish (2), Catalan (5), Italian (4) and the Laziale Italian variant (1). We classify these formulae, which lie on the thresholds of what strictly might be considered proverbs, in two groups: proverbs without meteorological implication and
\end{abstract}

${ }^{1}$ Nos remerciements s'adressent à Víctor Pàmies (Barcelone), Adriana Pintori (Barcelone) et Matteo Rivoira (Turin) pour leur aide précieuse et leur générosité en nous fournissant des renseignements.

2 Maria-Reina BASTARDAS RUFAT est Docteur en Philologie Romane et Maître de conférences à l'Université de Barcelone. Comme enseignante et chercheuse, elle s'est consacrée à la linguistique et, notamment, à la linguistique historique romane. Elle a travaillé en onomastique : toponymie (sujet de sa thèse) et anthroponymie (avec une participation active au projet PatRom). Actuellement, elle collabore au projet de recherche franco-allemand DÉRom (Dictionnaire Étymologique Roman). Elle a collaboré aussi au projet de recherche ParemioRom (Romance Paremiology: weather proverbs and territory) et est auteure de plusieurs publications sur la parémiologie. Courrier électronique : reina.bastardas@ub.edu.

3 Joan FONTANA I TOUS, Docteur en Philologie Romane de l'Université de Barcelone, il est Maître de conférences en langue roumaine, linguistique romane et littérature générale, et il assure, depuis 2004, des cours à ladite université. Ses travaux de recherche portent généralement sur la parémiologie comparative (langues romanes). Comme traducteur, il a offert au public catalan le roman Maitreyi de Mircea Eliade et, avec d'autres collègues, l'essai Amurgul gândurilor (Le crépuscule des pensées) d’Emil Cioran. Courrier électronique : joan_fontana@ub.edu.

${ }^{4}$ José Enrique GARGALLO GIL est Professeur de Philologie Romane à l'Université de Barcelone depuis 1982 et membre de la Section Philologique de l'Institut d'Estudis Catalans (IEC) depuis 2014. Il a organisé divers congrès sur différents thèmes, parmi lesquels on peut citer la parémiologie romane, sujet sur lequel il a dirigé des projets de recherche comme IP (Investigador Principal) entre 2005 et 2015. Il a participé à de nombreux congrès comme conférencier ou avec des communications. Il est auteur de plus de 300 publications, quelques-unes en collaboration. Sa dimension internationale s'enrichit avec divers séjours comme professeur visitant (Visiting Professor) : University of Illinois at Urbana-Champaign (1996), Saint-Petersburg (1997), San José de Costa Rica (1998) ou Université Stendhal de Grenoble (2004). Courrier électronique : gargallo@ub.edu. 
meteorological proverbs. On the other hand, we classify the examples of the second group in four sections according to the characteristics that are attributed to one or several months: proverbs with a characteristic for several months, proverbs with a characteristic for each pair of months, proverbs with a characteristic for some of the months, and sayings attributing a different feature for each month. This corpus offers a glimpse to the way Romance peoples view the months and it reveals affinities in the form of paremic types (or "paremiotypes"), which tell us about the popular culture shared by the peoples that are heirs of Rome.

Keywords: proverbs, month, year, meteorology, Romance languages.

REZUMAT. Proverbe romanice cu cele douăsprezece luni: caracterizarea paremiologică şi lunară a anului. În ţările romanice, există mai multe tipuri de proverbe meteorologice şi calendaristice. Nu numai în legătură cu motivaţia lor, dar, de asemenea, cu lungimea lor. Dintre proverbele care se referă la luni, câteva sunt foarte scurte: de pildă, așa-zisele „proverbe minime” au doar două cuvinte; mai sunt însă alte proverbe atât de lungi, încât, curios lucru, îngreunează orice fel de mnemotehnică. Tocmai din acestea din urmă fac parte proverbele corpusului nostru; este vorba, anume, despre treisprezece înşirări paremiologice referitoare la cele douăsprezece luni ale anului, care reprezintă cinci varietăţi romanice diferite; de la vest la est, portugheza (un proverb), spaniola (2), catalana (5), italiana (4) şi varianta italiană din Lazio (1). Aceste formule, care se află la limita a ceea ce am putea considera strict proverbe, se clasifică, întâi, în două grupe: proverbe fără nicio implicare meteorologică şi proverbe meteorologice. Pe de altă parte, grupăm exemplele din al doilea grup în patru capitole în acord cu caracteristicile care se atribuie la una sau la mai multe luni: proverbe cu o caracteristică pentru diferite luni, proverbe cu o caracteristică pentru câte două luni, proverbe cu o caracteristică pentru unele dintre luni şi proverbe cu o caracteristică pentru fiecare lună. Ansamblul oferă o privire romanică asupra cursului anului, care pune în evidență afinități sub forma unor „paremiotipuri” ale căror formule ne povestesc despre cultura tradiţională împărtăşită de acele popoare care sunt moştenitoarele Romei.

Cuvinte-cheie: proverbe, lună, an, meteorologie, limbi romanice.

\section{Introduction}

La renaissance longuement attendue de la nature arrive à son apogée pendant le mois de mai (pensons, par exemple, à l'exubérante floraison des cerisiers), 5 et est accompagnée d'un allongement de la journée, plus évident de

\footnotetext{
${ }^{5}$ Ce n'est pas par hasard que le nom populaire du mois de mai en roumain est florar (DEX: s. v. florar $^{2}$; 'Numele popular al lunii mai').
} 
jour en jour. Un délicieux proverbe, très court, résume cette période en roumain : Mai e Rai ['Mai est le Paradis'] (Rusu-Păsărin 2006 : 112). ${ }^{6}$ Voici une formule servant à exemplifier les deux caractéristiques formelles les plus importantes de n'importe quel proverbe : la brièveté et la rime, qui rendent plus facile la mémorisation. Dans ce sens, il est intéressant de noter que la sagesse populaire, fruit d'une expérience séculaire, ${ }^{7}$ peut s'exprimer en juste trois mots. On pourrait même raccourcir encore ce proverbe avec l'élision du verbe copulatif : *Mai, Rai, forme que, pourtant, nous n'avons pas réussi à documenter et qui rappellerait la structure du dicton roumain Dragoste, pacoste ['Amour, disgrâce'] (Zanne 2004 [1900] : VIII, 728, num. 15925), où le verbe $a$ fi est sous-entendu, et qui, par sa brièveté insupérable, ${ }^{8}$ entre dans la catégorie des proverbes minimaux. Par proverbes minimaux, nous désignons les structures parémiques à sens plein et qui sont formulées avec un verbe explicité ou bien élidé, et avec le nombre le plus petit possible de composants (v. Fontana 2019 : 38).

Si les proverbes minimaux sont surprenants par leur concision et leur formulation charmante, les longues formulations parémiques ne sont pas moins surprenantes, difficiles à mémoriser, avec une « forme très proche de la litanie ${ }^{9}$ (Montreynaud et alii 1993 : 106). Pour notre étude, nous prendrons un corpus de proverbes de ce dernier type : les proverbes qui mentionnent explicitement les douze mois de l'an.

Il faut dire, au préalable, qu'à l'inverse des proverbes minimaux, extrêmement et faciles à retenir, les formules qui font l'objet de cet article ne sont pas, à vrai dire, des proverbes, mais des formules ou litanies, ${ }^{10}$ qui, dans certains cas, réuniront quelques proverbes minimaux, déjà attestés ou qui, par leur forme, pourraient bien l'être (v. l'exemple 9). On ne doit pas s'étonner du

${ }^{6}$ Pour les proverbes romans du mois de mai, v. Gargallo (2018).

7 N'oublions pas que, en accord avec ce que Cervantes affirme, «los refranes son sentencias breves, sacadas de la experiencia y especulación de nuestros antiguos sabios » ['les proverbes sont des sentences courtes tirées de l'expérience et des observations de nos anciens sages'] (Quijote II, Cap. 67).

${ }^{8}$ Logiquement, une formule parémique à deux composants est la plus courte possible pour réaliser un rapport indiquant la caractéristique ou la conséquence entre les deux.

${ }^{9}$ Montreynaud et alii (1993 : 106), après avoir signalé que « La plupart du temps, on rencontre le dicton sous une forme courte ; mais, alors qu'il a l'air de se suffire à lui-même, il peut n'être qu'un fragment d'une forme plus longue », ajoutent un exemple qui est certes «spectaculaire »: "Année neigeuse, [/] Année fructueuse ; [/] Année nébuleuse, [/] Année plantureuse ; [/] Année venteuse, [/] Année pommeuse ; [/] Année sèche, [/] Année de vin ; [/] Année de glands, [/] Année cancéreuse ; [/] Année de champignons, [/] Année tourmentée ; [/] Année pluvieuse, [/] Année malchanceuse ; [/] Année de givre, [/] Année de fruits ; [/] Année de groseilles, [/] Année de bouteilles ; [/] Année de raves, [/] Année de santé ; [/] Année hannetonneuse, [/] Année pommeuse ; [/] Année de noisettes, [/] Année de disette».

10 Aussi, Montreynaud et alii (1993 : 106-107) signalent : "Le dicton pourrait ainsi être considéré comme une forme annelée, morcelable à loisir, un "lego" (jeu à éléments emboitables) de bouts rimés, que l'on a choisi concis et sec, ou alangui ». 
fait que les exemples recueillis proviennent de sources écrites, puisque on imagine difficilement un locuteur capable de réciter par cœur ces litanies.

En accord avec ce qui vient d'être dit, il n'est pas surprenant que le corpus réuni (onze exemples et deux variantes, 2a i 9a), soit plutôt réduit puisque, effectivement, ce type de formules, par leur extension et difficulté de mémorisation, est rarement présent dans les recueils de proverbes romans.

\section{Un proverbe sans allusion à la météorologie}

Depuis toujours, le génie populaire a su ajouter l'immédiat à la sagesse. Un bon exemple est l'astuce pour se rappeler du nombre de jours de chaque mois : on met de côté les deux poings, index contre index, et on commence à énumérer les mois par la jointure du petit doigt de la main gauche, qui fait une «bosse», et ainsi de suite en énumérant février dans le «creux» qui suit, mars dans la «bosse » suivante, etc. ; la «bosse» représente un mois à 31 jours, le " creux » à côté entre les deux jointures représente un mois court, à 30 jours (28 pour février). La jointure de l'index gauche est suivie par celle de l'index droit; deux «bosses » pour juillet et août, tous les deux à 31 jours. Cette astuce, bien à la portée de la main, est complémentaire des proverbes ou formules populaires bien connues telles que, en portugais, Trinta dias tem Novembro, Abril, Junho e Setembro, vinte oito terá um e os mais têm trinta e um ${ }^{11}$ (Machado 1998 : 612) ; en galicien, Trinta días ten novembro, con abril, xuño e setembro; vinteoito ou vintenove ten febreiro e os demais teñen trinta e un ${ }^{12}$ (Fraguas 2006 : 237) ; en espagnol, Treinta días trae noviembre, como abril, junio y septiembre; de veintiocho no hay más que uno; los demás, de treinta y uno ${ }^{13}$ (Doval 1997 : 372, num. 1873) ; ou l'italien Trenta dì conta novembre con april[,] giugno e settembre; di ventotto ce n'è uno, tutti gli altri n'han trentuno ${ }^{14}$ (Boggione, Massobrio 2007 : 10, num. I.3.1.16.II.). Le proverbe italien est si populaire qu'il apparaît

\footnotetext{
11 Machado ajoute des variantes comme :Trinta dias tem Novembro, Abril, Junho e Setembro; de vinte e oito só há um e os outros tem (os mais são de) trinta e um; ou bien: Trinta dias tem Setembro, Abril, Junho e Novembro, Fevereiro vinte e oito tem; se for bissexto mais um lhe dêem, $e$ os mais, que sete são, trinta e um todos terão. Tous les proverbes mentionnés dans ce paragraphe ont, à quelque variante minimale près, le sens de 'trente jours a novembre, avec avril, juin et septembre ; de vingt-huit on n'en a qu'un ; et le reste trente-un'.

12 On trouve encore sur internet (https://gl.wikipedia.org/wiki/Mes) la variante : Trinta días ten novembro, [/] con abril, xuño e setembro, [/] con 28 hai só un, [/] e o resto trinta e un.

13 Doval recueille une autre variante : Treinta días trae noviembre con abril, junio y septiembre; veintiocho trae uno, y los demás, treinta y uno.

14 Voici encore deux variantes recueillies par Boggione et Massobrio sur la même page : Trenta dì ha novembre, april, giugno e settembre; di ventotto ce n'è uno [;] tutti gli altri n'han trentuno (num. I.3.1.16); et Trenta dì ha novembre, aprile[,] giugno e settembre; di ventotto ce n'è uno; tutti gli altri son trentuno (num. I.3.1.16.I.).
} 
dans le titre du recueil d'Antoni et Lapucci (1993) intitulé 30 dì conta novembre... Un seul cas isolé, parmi les proverbes appartenant à cette catégorie parémique, comporte une formulation qui mentionne explicitement tous les douze mois ; c'est le proverbe catalan :

(Exemple 1) Trenta dies té setembre, amb abril, juny i novembre. Els altres de trenta-u[,] gener, marc, maig, juliol, agost, octubre i desembre, menys febrer amb vint-i-vuit (Sabaté, Culla 1994 : 43).

\section{Proverbes météorologiques}

Par la suite, nous présenterons les proverbes qui, comme le précédent, contiennent les douze mois de l'année explicités dans leur formulation, mais qui, à la différence de l'exemple 1, ont une composante météorologique (au sens large ; phénomènes météorologiques mais aussi travaux agricoles, saison des fruits, etc., qui sont, en dernier terme, en rapport avec le climat). Pour rendre plus facile leur caractérisation, nous les avons arrangés en quatre groupes: ceux qui indiquent une seule caractéristique pour plusieurs mois (3.1.), ceux qui en indiquent une pour chaque paire de mois (3.2.), ceux qui en indiquent une pour quelques mois (3.3.) et ceux qui en indiquent une pour chaque mois (3.4.).

\subsection{Avec une seule caractéristique pour plusieurs mois}

Pour les deux litanies suivantes correspondant au catalan, l'exemple $2 a$ n'est qu'une variante raccourcie de l'exemple 2 . En réalité, les trois seules différences sont que l'exemple $2 \mathrm{a}$ introduit le pronom personnel de première personne singulier au troisième hémistiche, qu'il substitue le castillanisme adiós au catalan adéu dans le quatrième hémistiche, et qu'il omet absolument le cinquième.

(Exemple 2) Octubre, novembre, desembre, [/] ${ }^{15}$ gener, febrer, marc, abril, maig, [/] juny, juliol, me'n vaig; [/] adéu, agost i setembre, [/] $i$ adéu l'any, perquè mén vaig (Amades 1982 : V, 274 ; aussi dans Parés 1999 : 73, num. 521, qui l'écrit sans césures).

['Octobre, novembre, décembre, [/] janvier, février, mars, avril, mai, [/] juin, juillet, je m'en vais [/] adieu, août et septembre, [/] et adieu à l'année, puisque je m'en vais']

(Exemple 2a) Octubre, novembre, desembre, [/] gener, febrer, marc, abril, maig, [/] juny, juliol, jo me'n vaig [/] adiós agost i setembre (Bonafè 2009: 42).

\footnotetext{
${ }^{15}$ Avec la barre oblique entre crochets nous signalons un retour à la ligne dans la source.
} 
Il faut avouer que le sens de ces proverbes n'est pas clair. Conscient de ce fait, Amades, en recueillant l'exemple 2, ajoute le commentaire : «La pagesia conserva un refrany que, segons interpretació rural, presenta el curs de l'any començant per l'octubre, de quan s'iniciava pel sembrar» ['Les paysans conservent un proverbe qui, d'après l'interprétation rurale, présente le cours de l'an débutant par le mois d'octobre, quand les semences commençaient']. De sa part, Parés (1999: 73, num. 521) parachève avec la remarque « Aquest calendari només val per a comarques cerealístiques però no vinateres, perquè el setembre seria un mes de molta feina " ['Ce calendrier ne vaut que pour les contrées céréalistiques mais non pour les viticoles, où septembre est un mois de travail intense']. Le début de l'an après la fin de l'été doit être mis en rapport avec le nom du mois de septembre en sarde, cabidani ou cabudanni < CAPUT ANNI 'tête de l'an' (AIS 324), et, peut-être, avec le début de l'an hébreu, qui a lieu à la première lune de l'automne (v. Tagliavini 1963 : 160).

\subsection{Avec une caractéristique pour chaque paire de mois}

(Exemple 3) Gennaio e febbraio, tienti al pollaio; marzo e aprile, capretto gentile; maggio e giugno, erbette col grugno; luglio e agosto, piccioni arrosto; settembre e ottobre, buone lepri col savore [sauce]; novembre e dicembre, buon vitel sempre (Boggione, Massobrio 2007: 10, num. I.3.1.6; 206, num. V.1.7.1.19).

['Janvier et février, occupe-toi du poulailler; mars et avril, chevreau tendre ; mai et juin, des herbes à cœur (c.-à-d. artichauts, etc.) ; juillet et août, pigeons rôtis; septembre et octobre, bon lièvre avec la sauce ; novembre et décembre, bon veau toujours']

Il est intéressant de remarquer que l'exemple 3 est assez connu et apparaît dans les trois sources italiennes que nous avons consultées (Schwamenthal, Straniero 1991; Boggione, Massobrio 2007 et Lapucci 2007), à quelques différences près : chez Schwamenthal, Straniero (1991: 257, num. 2801) et Lapucci (2007 : 659, num. 405), avec tieniti et polli e piccioni au lieu de tienti et piccioni, respectivement. En outre, Schwamenthal, Straniero donnent vitello buono, au lieu de buon vitel, tandis que Lapucci propose les deux possibilités. Finalement, ce dernier auteur divise le proverbe en douze vers.

Entrant dans le sens du proverbe, pendant janvier et février il faut être attentif au poulailler puisque, après la période hivernale, les poules recommencent à pondre et il faut remplacer celles qui ne pondent plus. Deux proverbes minimaux, en italien et galicien, nous renseignent sur cette période : Gennaio 
ovaio $^{16}$ (Schwamenthal, Straniero 1991 : 258, num. 2809 ; Boggione, Massobrio 2007 : 11, num. I.3.2.1.34 ; 91, num. II.9.7.35 ; Lapucci $2007: 658$, num. 399) et Xaneiro, oveiro (Vázquez 2003 : 99, num. 2375). Les mois de mars et avril nous offrent des agneaux et chevreaux tendres (c'est le sens de gentile). Au mois de mai et juin, on récolte les meilleurs légumes, ceux qui ont grugno, mot qui apparait, à cause de la rime, au lieu de grumolo, c'est à dire, le cœur intérieur tendre. Pendant les mois de juillet et août, on a de nouveaux produits du poulailler et du pigeonnier. Pour ce qui est du gibier, les mois propices sont septembre et octobre : les lièvres avec savore, mot générique pour désigner une sauce aigre-douce, dont on connaît plusieurs variantes en Italie, avec ingrédients différents, et qui va avec le gibier.17 Finalement, pour novembre et décembre, on conseille la viande de bœuf qui, même si elle n'a pas une saison propice, convient pour passer les rigueurs de l'hiver.

Pour clore ce chapitre nous présentons une variante des Abruzzi italiens, que nous n'avons pas inclus dans notre corpus puisque la mention explicite des deux derniers mois manque :

Ggennar' e frebbare,/la halline n'n à pare;/marz' e aprile,/agnell' e crapette ggendile;/magg' e ggiugne,/vetell' a lu grugne;/ lujj' e ahoste,/pullastr' arroste;/settembr' e uttobre,/presutt' a ll'ombre; /lu reste de ll'avetre mese,/hallenacc' e pporc' appese (Schwamenthal, Straniero 1991: 257, num. 2801) ['Janvier et février,/les poules sans égal ;/mars et avril,/agneaux et chevreaux tendres; /mai et juin, /veau avec son museau ; 18 /juillet et août,/poulets rôtis;/septembre et octobre, /le jambon à l'ombre;/le reste des autres mois,/dindon et porc pendu'].

Il est intéressant de remarquer que, par rapport à l'exemple 3, il y a des divergences pour trois paires de mois : le veau est le protagoniste de mai-juin, le séchage du jambon l'est en septembre-octobre, et l'incontournable abattage du porc et le dindon (hallenacc $[e]$ ) de Noël sont les protagonistes de novembre-décembre.

\subsection{Avec une caractéristique pour quelques mois}

(Exemple 4) Molt fred sol ésser el gener [/] i el febrer per lo semblant; [/] pel març plou de tan en quan, [/] per l'abril s'aigo ja cau bé, [/] pel maig m'aparellaré, [/] pel juny a segar aniré, [/] i pel juliol a batre; [/] d'agost figues menjaré [/] per entrar gras a setembre, [/] d'octubre pas a novembre [/] i a desembre acabaré [/] i gràcies a Déu faré [/] com he

\footnotetext{
16 Selon la glose de Lapucci, « dopo un periodo di stasi le galline riccominciano a fare le uova in abbondanza ».

${ }^{17} \mathrm{Au}$ nord-est de l'Italie (Trentino Alto Adige, Veneto et Friuli Venezia-Giulia), par exemple, elle était élaborée avec des oignons, vinaigre ou jus de citron, raisins secs et pignons (v. Treccani : s. v. savóre).

18 Le sens de cette expressión n'est pas clair.
} 
passat aquest any [/] i si no tenc ningun dany [/] un altre en començaré (Bonafè 2009: 42).

['Très froid est souvent janvier [/] et février pareillement ; [/] en mars il pleut de temps à autre, [/] en avril l'eau tombe bien, [/] au mois de mai je serai en couple, [/] au mois de juin j'irai à la moisson, [/] au mois de juillet à battre, [/] en août je mangerai des figues [/] pour entrer bien gras en septembre, [/] d'octobre on passe à novembre [/] et décembre je finirai [/] et je remercierai Dieu [/] puisque j'ai passé cette année [/] et si je n'ai pas de mal [/] une autre en recommencerai']

Cette litanie parémique catalane rappelle un proverbe bien usuel dans cette langue : Qui dia passa, any empeny (PCCD) ['Qui passe un jour, pousse un an'] ; c'est à dire, qui sait résoudre les difficultés de chaque jour, jour à jour, arrive à résoudre celles de toute l'année. Remarquons que les derniers quatre mois de l'année, septembre, octobre, novembre et décembre, sont mentionnés sans aucune caractéristique agricole ou météorologique, sauf pour le conseil d'entrar gras a setembre, pour surmonter sans peine les rigueurs des mois d'automne et d'hiver.

En reprenant notre exemple dès le début, il faut rappeler, par rapport au froid de canard attribué au mois de janvier, que, dans le domaine catalan et espagnol, la période la plus froide de l'an correspond à la semaine comprise entre le 15 janvier (Saint Paul Ermite ou de Thèbes, et Saint Maur) et le 22 janvier (Saint Vincent). On appelle cette semaine la «setmana dels sants barbuts » ['semaine des saints barbus'], dénomination fondée sur l'iconographie traditionnelle de Saint Paul Ermite, Saint Maur, et Saint Antoine le Grand ou du Désert (17 janvier ; connu en catalan comme «Sant Antoni Abat» ou «Sant Antoni dels ases » ['des ânes'] ou «del porquet» ['du pourcelet'] à cause de son patronage sur les animaux domestiques). Pour Saint Vincent, il n'est pas traditionnellement représenté avec une barbe, mais il clôt quand même la froide semaine des saints barbus. D'un autre côté, notre exemple fait durer le froid de janvier jusqu'au mois suivant; ce n'est pas par hasard que ce proverbe italien propose ce conseil pour les vêtements (v. l'exemple 10) pour les deux mois : Gennaio e febbraio metti il tabarro (Lapucci 1995 : 207) ['Janvier et février, met le manteau'].

Par la suite, mars et avril sont caractérisés par la pluie in crescendo: pel març plou de tan en quan, [/] per l'abril s'aigo[19] ja cau bé. ${ }^{20}$ Effectivement,

\footnotetext{
19 À noter la variante dialectale monophtonguée aigo (aigua, en catalan normatif), correspondant au catalan des Baléares.

20 V. l'italien Marzo piovi, piovi, [/] april non cessar mai, [/] maggio dammene una che n'ho assai (Antoni, Lapucci 1993 : 86) ['Mars, il pleut, il pleut, [/] avril n'arrête jamais, [/] mai donne-moi seulement une [pluie] et ça suffit'].
} 
comme pour le catalan, en portugais Em Março chove cada dia um pedaço (Moreira 2003 : 107, num. 292) ['Au mois de mars il pleut chaque jour un peu'] ; et il vaut mieux que ça soit ainsi puisque, si la pluie est trop intense au mois de mars, elle est nuisible comme le prouvent des proverbes partout dans la Romania : en asturien, Marzu de lluvies cargau, fai al añu disgraciau (Castañón 1962: 200); en espagnol, Marzo de lluvias cargado, año muy desgraciado (Martínez Kleiser 1945 : 209) ; en catalan, Març molt plujós, / any dolorós (Sanchis 1951 : 53, num. 59) ; en français, Mars pluvieux, [/] An disetteux (Cellard, Dubois 1985 : 38) ; en romanche, Marz plova, la terra dola (Hauser 1975 : 617) ; ou, en frioulan, Se març ti bagne no sperâ grande cucagne (Del Fabro 2000 : 145).

Pour mettre en relief la valeur des pluies d'avril, ${ }^{21}$ il suffira de rappeler deux proverbes qui mentionnent deux des grands rois de l'Ancien Testament (David et Salomon, respectivement, père et fils) : Valen máis as augas de abril có carro do rei David22 (ALGa 2003 : IV, 462, num. 16), en galicien; et Le dolci acque d'aprile valgon più che il trono di Salomone ${ }^{23}$ (Antoni, Lapucci 1993 : 107), en italien. C'est vrai que, comme on dit en portugais et en règle générale, Nunca a chuva de Abril é mau tempo (Carrusca 1976 : III, 218). En ce sens, un avril pluvieux est un excellent présage pour la récolte: Abril molhado, ano abastado (Ferreira 1999: 216), en portugais ; Abril llovedero llena el granero (Martínez Kleiser 1945 : 221), en espagnol ; Abril plujós, / graner abundós (Sanchis 1951 : 64, num. 32), en catalan ; Plueio d'abriéu emplis lou granié (Mistral 1979 : II, 84, s. v. granie), en occitan; April piovoso, anno ubertoso (Schwamenthal, Straniero 1991 : 42, num. 444), en italien ; ou Avrîl ploiôs, an bondançôs (Martinis 2010 : I, 63), en frioulan. En somme, on peut sans doute dire que Pluie d'avril, [/] trésor du pays (Hauser 1975 : 327).

Suit une affirmation un peu surprenante : Maig m'aparellaré. Il nous semble qu'il ne s'agit pas d'un mariage, puisque, comme le proverbe dit, Bodes maials, bodes mortals (Parés 1999: 119, num. 2159) ['Noces en mai, noces mortelles'], mais la période où les moutons sont en rut, élément fondamental pour l'économie des bergers. Effectivement: Pel maig, cada ovella amb sa parella (Parés 1999: 122, num. 2285) ['Au mois de mai, chaque brebis avec son partenaire'] et, en conséquence, Bels de maig, belets d'octubre (Parés

21 On pense aux proverbes onomatopéiques Arvì, ciuvì, ciuvì ['Avril, il pleut, il pleut'] (Schwamenthal, Straniero 1991 : 44, num. 460), piémontais, et Arvì, [/] ciuì ciuì (Lapucci 1995 : 212), ligure, qui évoquent le tambourinage des gouttes de pluie pendant ce mois.

22 'Les eaux d'avril valent plus que le char du roi David'; voir l'Atles de ParemioRom pour ce parémiotype. Localisé à Vilarín do Castelo (municipalité de A Fonsagrada, province de Lugo).

23 'Les douces eaux d'avril valent plus que le trône du roi Salomon'. Avec la glose : «Per quanta acqua venga d'aprile nessuna cosa della campagna potrà soffrirne » (Antoni, Lapucci 1993 : 108) ['Peu importe combien d'eau tombe en avril, elle ne sera jamais nuisible aux récoltes']. 
1999 : 119, num. 2158), proverbe charmant qui met en rapport les bêlements de mai avec les faibles bêlements des agneaux en octobre.

Pour ce qui est du mois de juin, nous citons le commentaire d'Amades :

\begin{abstract}
Aquest mes [juny] marca un dels moments més importants i més culminants dins de la vida dels pobles de cultura mediterrània, en els quals el blat i els cereals en general juguen el paper principal d'alimentació i on fins a temps moderns llur escassetat o abundor ha estat la clau de la fam o del benestar. El juny és el temps de les messes (Amades 1983 : III, 693)

['Ce mois [juin] est un des moments les plus importants et décisifs pour la vie des peuples à culture méditerranéenne, pour lesquels le blé et les céréales en général ont un rôle très important dans l'alimentation et où, jusqu'aux temps modernes, le manque ou abondance de céréales ont été la clé pour une année de disette ou bien-être. Juin est le temps des moissons']
\end{abstract}

Dans l'ouvrage d'Amades, cette explication précède un des proverbes les plus connus du catalan : Pel juny [/] la falç al puny ['Au mois de juin, la faucille au poing'], ${ }^{24}$ qui s'accorde avec la formule de notre exemple : pel juny $a$ segar aniré, ${ }^{25}$ ou avec l'expression portugaise Junho ceifar de l'exemple 6 (nous renvoyons à cet exemple pour plus de détails ; v. aussi Correas, Gargallo 2003 : 200). La moisson de juin précède le battage de juillet, ${ }^{26}$ pel juliol a batre [aniré], puisque la température de ce mois " és la millor de tot l'any per a batre, perquè sol ésser molt seguida i regular: al matí, vent serè, que ajuda i facilita la rompuda i batuda de les espigues; al migdia, sol fort, que torra la batuda; cap al tardet, marinada, que facilita la feina del ventar i de separar el gra del boll $»^{27}$ (Amades 1983 : IV, 377) ['est la meilleure pour battre puisqu'elle est régulière; au matin du vent qui aide à battre les épis ; à midi, fort soleil, qui grille la gerbe ; au soir, vent marin, qui facilite la tâche de vanner et séparer le grain de la balle']. De cette croyance, proviennent des proverbes comme le catalan $\mathrm{Al}$

\footnotetext{
${ }^{24}$ Amades clôt avec l'affirmation : «Hom pot dir que tota la vida rural dels pobles del migdia europeu gira a l'entorn del concepte expressat per aquesta parèmia » ['On peut donc dire que toute la vie rurale des peuples du midi européen tourne autour de l'idée exprimée par ce proverbe'].

25 Selon le proverbe catalan, Sembraràs quan voldràs, [/] però pel juny segaràs (Amades 1983 : III, 698) ['Tu peux semer quand tu veux, mais tu feras la moisson en juin'].

${ }^{26}$ Aussi l'importance accordée à il buon batter di luglio dans ce proverbe toscan : Il gran freddo di gennaio, il mal tempo di febbraio, il vento di marzo, le dolci acque di aprile, le guazze di maggio, il buon mieter di giugno, il buon batter di luglio, le tre acque d'agosto con la bona stagione, vagliono più che il tron di Salomone (Bellonzi 2000 : 121, num. 1602).

27 Sans doute, « La vida pagesa d'aquest moment de l'any [juliol] està completament absorbida pel batre » (Amades 1983 : IV, 369) ['La vie des paysans à ce moment de l'année [juillet] est absolument prise par le battage'].
} 
juliol, bat qui vol (PCCD) ou le portugais Nos dias de Julho, [/] Eu ceifo e debulho; [/] Se o vento vai dando, [/] Vou logo ensacando (Carrusca 1976 : III, 233) (v. l'exemple 6).

Finalement, manger des figues au mois d'août, d'agost figues menjaré, est bien logique puisque la saison de ce fruit arrive pendant ce mois; les proverbes suivants nous renseignent en catalan, occitan et gascon : A l'agost, figues i most ${ }^{28}$ (Parés 1999: 139, num. 3025), En avoust, [/] figo e moust (Mistral 1979 : I, 198, s. v. avoust), et Au mes d'aost, higas e most (Armanac Gascon 1985 : 124) [tous avec le sens de 'en août, figues et moût'].

(Exemple 5) En enero, el gato en celo; febrero, merdero; marzo, sol como mazo; en abril, aguas mil; en mayo, toro y caballo; en junio, hoz en puño; en julio, calentura y aúllo; en agosto, frío en rostro; en setiembre, el rozo y la urdiembre; en otubre uñe los bueyes y cubre; en noviembre y deciembre, coma quien tuviere, y quien no tuviere, siembre (Correas 2000 [1627] : 315, num. 1635).

['En janvier le chat en rut ; février, rabougri ; mars, le soleil comme une massue ; en avril, mille eaux; au mois de mai, taureau et cheval; en juin, la faucille au poing ; en juillet, chaleur jusqu'à hurler ; en août, froid sur le visage ; en septembre, labourer et filer ; en octobre atteler les bœufs et couvrir les champs semés ; en novembre et décembre, que mange qui en a, et celui qui n'en a pas, qu'il sème'].

L'exemple 5 apparaît dans Martínez Kleiser (1995 : 468, num. 40.910), qui renvoie à l'ouvrage de Correas de 1627, que nous citons. Le proverbe est mentionné, donc, dans une source du premier tiers du XVIIème siècle, c'est à dire que le proverbe existerait déjà dans l'Espagne de Cervantes et du Quijote et qu'il fournirait un schéma pour caractériser les douze mois de l'an. Voyons ce qu'il attribue aux différents mois. Le premier élément, En enero, el gato en celo, rappelle des proverbes catalans assez fréquents comme Gat miolador, [/] gener a la porta ['Chat qui miaule, [/] le mois de janvier est à la porte'] ou Pel gener [/] els gats cerquen muller ['Au mois de janvier [/] les chats cherchent épouse'] (Amades 1982: I, 291). Quant à febrero, merdero, la note 200 de l'ouvrage de Correas explique : «Merdero* : porque es el más corto de los doce meses, como si hubiera crecido menos que sus hermanos » ['Merdero parce que c'est le plus court des douze mois, comme s'il avait grandi moins que ses frères'] ; effectivement, merdero est un nom assez mal attesté (il n'apparaît pas dans le $D L E$ ou le $D C E C H$; CORDE cite uniquement Correas) mais qui désigne

\footnotetext{
28 Pour plus d'exemples et variantes de ce proverbe, v. la $P C C D:<$ https://pccd.dites.cat/?paremiotipus= $\mathrm{A}+1 \% 27$ agost $\% 2 \mathrm{C}+$ figues $+\mathrm{i}+$ most $>$.
} 
le plus petit et faible animal d'une portée. ${ }^{29}$ Pourtant, le fait que le mois de février soit plus court n'empêche pas qu'il soit redoutable: Février, le plus court des mois, [/] Est de tous le pire à la fois (Cellard, Dubois $1985: 23$ ), en français ; Febrero corto, el peor de todos (Martínez Kleiser 1995 : 470, num. 41.065), en espagnol ; Febbraiuzzo, peggio di tutto (Schwamenthal, Straniero 1991 : 243, num. 2636), en italien ; ou Fevrarut piês di dut (Ostermann 1995 : 30), en frioulan. La caractérisation du mois de mars, marzo, sol como mazo, est fondée sur la paronymie marzo - mazo, que nous retrouvons dans le proverbe Sol de marzo / pone la cabeza como un mazo, localisé à Teruel par Gomis (1998: 159, note 23) ; de même, les conséquences du soleil de mars se manifestent dans les proverbes suivants : El sol de març crema la cara ['Soleil de mars brûle le visage'] (DCVB : s. v. sol), en catalan ; A lu sôli marsulinu, [/] na dà capu, ne dà spìn ['Au soleil de mars, [/] ni le visage ni le dos'] (Sampieri 1996 : 154), en corse ; Su sole de martu ponet su marcu ['Le soleil de mars, laisse sa marque', c'est à dire, il fait mal à la tête] (Spano 1997 : 220, s. v. Martu), en sarde. Un parémiotype ibérique nous renseigne sur le soleil qui brûle même les dames dans leurs palais : Sol de Março, queima a dama no Paço (Reis 1995 : 83, 207, 215, 255) en portugais ; Sol de març / crema les dames a palau ${ }^{30}$ (Sanchis 1951 : 52, num. 30), en catalan; et En marso o sol queima a dama no palacio ${ }^{31}$ ( $A L G a$ 2003 : IV, 465, num. 190), en galicien.

Pour ce qui est d'en abril, aguas mil, nous renvoyons à l'exemple 4 et notons en passant trois proverbes espagnols: Abril abrilero, cada día dos aguaceros (Martínez Kleiser 1995 : 471, num. 41.202) ['Avril «avrilier», chaque jour deux averses', en rime en espagnol], Abril, para ser abril, ha de tener aguas mil (num. 41.205) ['Avril, pour être vraiment avril, doit avoir mille eaux'], et Abril, aguas mil, si no al principio, al medio o al fin (num. 41.203) ['Avril, mille eaux ; si ce n'est pas au début, ça sera au milieu ou à la fin']. Pour comprendre la formule en mayo, toro y caballo, il faut rappeler que ce mois est le mois le plus populaire des "corridas» et, pour ce qui est des chevaux, En mayo, cuando los grandes calores, los caballos están gordos y los potros corredores (Martínez Kleiser 1995 : 91, num. 8.147) ['Au mois de mai, avec les grandes chaleurs, les chevaux sont gras et les poulains coureurs'] et Caballo, por mayo; y eso, si me lo hallo (num. 8.148). Pour en junio, hoz en puño, nous renvoyons à

\footnotetext{
${ }^{29}$ V. «Merdero: pollo... atrasado en su crecimiento; canijo. "Este pollo es el merdero de la última echadura"» (Alcalá Venceslada, Voc. andaluz). «Hebrero merdero, un rato malo y otro bueno». V. aussi le proverbe minimal galicien: Febreiro, merdeiro (Rúa 2014: 178), localisé dans la zone du Bierzo (province de León).

${ }^{30}$ Localisé à Cadaqués (province de Girona).

31 Localisé à A Serra de Outes (province de A Coruña). La notation marso, au lieu de marzo, est due au « seseo » qui caractérise cette zone.
} 
Rodríguez (1896: 106), avec le même proverbe mais sans la préposition initiale, et aussi à l'exemple 6. D'un autre côté, en julio, calentura y aúllo est en rapport avec la chaleur typique de ce mois ; ce n'est pas par hasard qu'on dit : En julio beber y sudar, y el fresco en balde buscar (Martínez Kleiser 1945 : 266) ['En juillet on boit et on sue et on cherche le frais en vain']. En passant au mois d'août, en agosto, frío en rostro, Martínez Kleiser (1945: 272) propose deux proverbes paronymes : Agosto, frío en rostro ${ }^{32}$ et Agosto, fríe el rostro ; cet apparent contre-sens s'explique par le contraste entre nuit et jour : En agosto, de día fríe el rostro y por la noche, frío en el rostro33 (Pejenaute 1999 : 219).

D'un autre côté, en septembre il faut préparer la terre pour la semence (rozar, acception $1 \mathrm{du} D L E$ ) et, avec la diminution progressive des heures de lumière par jour, s'approche le temps pour filer. Le conseil pour octobre est en rapport avec la semence des céréales et des plantes fourragères : En octubre toma los bueyes y cubre (Martínez Kleiser 1995 : 18, num. 1.599) ['En octobre prend les bœufs et couvre les champs semés']. La litanie se clôt avec une formule moralisante : en noviembre y deciembre, coma quien tuviere, y quien no tuviere, siembre (v. le portugais Novembro semear, de l'exemple 6).

\subsection{Avec une caractéristique pour chaque mois}

Indépendemment du nombre de jours du mois, les proverbes considèrent chaque mois comme une unité et, avec pragmatisme, ils leur donnent une seule caractéristique (un météore, la saison d'un fruit, céréale ou animal...) que l'on considère constante pendant les $28,29,30$ ou 31 jours du mois. C'est pour cette raison qu'on trouve des traditions comme les cabañuelas en Espagne (pour lesquelles Gargallo 1999: 236-238); il s'agit d'une prédiction basée sur l'observation des phénomènes météorologiques pendant douze jours, par exemple, ceux entre la Sainte Lucie (13 décembre) et Noël (25 décembre) ou de Noël à l'Epiphanie ( 6 janvier), et qu'on croit qu'ils préfigurent les douze mois (le temps des douze jours correspond au temps qu'on aura chacun des douze mois). Cette prédiction des douze jours pour les douze mois reprend le mythe de l'éternel retour de Mircea Eliade, puisque les prédictions des douze jours servent à créer les douze mois à venir. ${ }^{34}$

\footnotetext{
32 'Août, froid sur le visage'. L'autre proverbe peut se traduire par 'Août, fait frire le visage'. Pour des proverbes parallèles en portugais, galicien ou asturien, v. Correas, Gargallo (2003 : 231).

33 Pour le retour des premiers froids : Primer día de agosto, primer día de invierno (Martínez Kleiser 1945 : 272) ['Premier jour d'août, premier jour d'hiver'].

34 Semblablement, les paysans catalans ont encore aujourd'hui l'habitude de faire, le 31 décembre, le calendari de la ceba 'calendrier de l'oignon' : « Tallen una ceba i en fan dotze clofolles, una per a cada mes, i les posen arrenglerades per tal d'establir quin mes representa cada una,
} 
Dans ce sens, notre corpus réunit sept proverbes qui attribuent une particularité à chacun des douze mois : trois proverbes italiens, (exemples 9, 10 et 11), et un espagnol (exemple 7), un catalan (exemple 8), un dans la variante italienne du Lazio (exemple 9a) et un portugais (exemple 6). Nous commençons par celui-ci :

(Exemple 6) Outubro revolver, Novembro semear, Dezembro nascer; nascer um Deus para nos salvar; Janeiro gear, Fevereiro chover, Março encanar, Abril espigar, Maio engrandecer, Junho ceifar, Julho debulhar, Agosto engravelar, Setembro vindimar (Machado 1998 : 438).

['Octobre, labourer ; novembre, semer ; décembre, naître ; naître un Dieu pour nous sauver; janvier, geler ; février, pleuvoir ; mars, construire des canalisations avec des cannes ; avril, épier ; mai, grandir ; juin, faucher ; juillet, battre ; août, faire des gerbes ; septembre, vendanger']

Comme nous l'avons déjà noté (3.1.), le début de l'année agricole est en octobre ; c'est pour cette raison que l'exemple 6 débute par octobre et non pas par janvier. Quant aux deux premiers mois mentionnés, les proverbes correspondants existent comme proverbes minimaux: Outubro, revolver (Machado 1998: 438) et Novembro, semear (1998: 382). De son côté, le mois de décembre est le seul caractérisé par une commémoration religieuse et non pas par un travail agricole ou une caractéristique météorologique, la naissance du Christ : Dezembro, nasceu Deus para nos salvar (Machado 1998 : 193). Pour ce qui est de la caractéristique la plus remarquable de janvier, partout dans la Romania c'est le froid : on n'a qu'à penser au roumain Călindari[35]/Cu gheţuri mari ['Janvier/ Avec les grandes gelées'] (Rusu-Păsărin 2006 : 12), et jusqu'à l'asturien En Xineru cuaya'l agua'n pucheru (Castañón 1962 : 159) ou l'espagnol En enero, / se hiela el agua en el puchero ${ }^{36}$ (ALeCMan 2003: 905, Notas : Enero ; très bien représenté dans la région de Castille-La Manche). Pour cette même période hivernale de l'an, on

segons l'ordre. Dins de cada clofolla tiren un polset de sal. L'endemà [primer dia de l'any] hom observa amb atenció fins a quin grau s'ha fos la sal. Les clofolles que la presenten molt dissolta assenyalen mesos humits i aquells en què la sal es manté sense fondre's indiquen mesos eixuts i de poca pluja » (Amades 1982 : I, 277) ['On prend un oignon et on le coupe en quarts, on prend douze tranches, une pour chaque mois, en mettant un peu de sel dans la concavité. Le lendemain (1er janvier) on observe le point d'humidité ou dissolution du sel sur chaque partie d'oignon ; les tranches qui gardent le sel plus sec sont l'augure d'un mois sec, celles où le sel est dissous sont l'augure d'un mois humide'].

35 Călindari, du latin CALENDARIU, dérivé de CALENDAE, est la dénomination populaire du mois de janvier (DEX: s. v. cărindar) ; ianuarie dans la langue normative.

${ }^{36}$ Les deux, au sens de 'en janvier l'eau gèle dans les casseroles'. Aussi en espagnol on dit : Enero, claro y helero (Martínez Kleiser 1995 : 474, num. 41.451) et aussi en catalan Gener, I fred i gelader (Sanchis 1951 : 12, num. 11). 
trouve deux parémiotypes : un indique que les pluies de décembre portent des gelées en janvier: Dezembro molhado, Janeiro geado (Reis 1995: 203), en portugais; Diziembre mojau, enero bien chelau (Palacios, Baos 2010 : 168, num. 70), en aragonais ; ou Desembre mullat, / i gener ben gelat (Sanchis 1951 : 144, num. 6), en catalan. D’après l'autre, les fortes gelées de janvier bâtissent des ponts (de glace sur les ruisseaux) que le mois de février peut rompre avec le dégel; nous en trouvons des exemples en occitan albigeois, francoprovençal d'Italie, italien et romagnol : Janviè fa lou pount [/] E febriè lou roump (Mistral 1979 : II, 154, s. v. janvié) ; Janvië feit lo pon; Fevrë lo ront (Cassano 1988 : 44, num. 151) ; Gennaio fa il ponte e febbraio lo rompe (Antoni, Lapucci 1993 : 46) ; et Znêr e' fa e' pont e fabrêr u'l romp (Spallicci 1996 : 64, num. 473). En reprenant notre proverbe portugais, on trouve dans Machado (1998: 278) les proverbes minimaux suivants : Janeiro, gear (qui coïncide, sauf pour la virgule, avec le texte de la litanie) et Janeiro geadeiro ${ }^{37}$; les deux rappellent les galiciens Xaneiro xeadas ${ }^{38}$ (ALGa 2003 : IV, 462, num. 24) ou Xaneiro xeadeiro ${ }^{39}$ (ALGa 2003 : IV, 462, num. 27).

Pour ce qui est de février, le portugais Fevereiro, chover (Machado 1998 : 246) coïncide avec d'autres proverbes minimaux romans, comme le galicien Febreiro moliceiro ${ }^{40}$ ['humide'] (ALGa 2003 : IV, 465, num. 186) ; l'occitan (de Bordeaux) Febrèi [/] Plugèi (Mistral 1979: I, 1110, s. v. febrié); ou le catalan Febrer, / plover (Sanchis 1951: 33, num. 36). Les bénéfices de la pluie de février sont visibles dans les deux parémiotypes suivants ; le premier compare la pluie de février avec le bon fumier : Chuva de Fevereiro vale por estrume (Machado 1998: 147), en portugais; Lluvia de febrero, el mejor estercolero (Sbarbi 1980 : 299), en espagnol ; Una piogia de Fevrè [/] A val lo seuscht dou feumé (Cassano 1988: 19, num. 51), en francoprovençal d'Italie ; ou Eau de février, [/] Eau de fumier (Cellard, Dubois 1985 : 24), en français. Pour ce qui est du

37 Ce proverbe minimal peut être complété par cette prédiction :

Janeiro - geadeiro,/Sobe-te ao outeiro:/Se vires verdejar,/Põe-te a chorar;/Se vires esterregar[]/Põete a cantar ['Janvier qui glace,/Monte sur une montagne :/Si tu vois verd,/Tu peux pleurer ;/Si tu vois terreux,/Tu peux chanter']

(Leite de Vasconcelos 1994-2007 : VIII, 32) ; si on voit verd déjà en janvier, les plantes poussent trop tôt et elles seront abîmées par le froid qui viendra encore.

38 Localisé à Meira (province de Lugo).

39 Un janvier xeadeiro est la première condition pour une bonne récolte, comme indique le proverbe galicien Con xaneiro xeadeiro, febreiro nevareiro, marzo sollarzo, abril mollado, maio pardo, san Juan claro valen máis que tus mulas y tu carro (ALGa 2003: IV, 462, num. 1; localisé à Constantín, municipe de Baralla, province de Lugo). Au même sens, le portugais Janeiro geoso, Fevereiro nevoso, Março molinhoso, Abril chuvoso e Maio ventoso, fazem o ano formoso (Machado 1998 : 278) ; l'espagnol Enero heloso, Febrero hebroso, Marzo airoso y Abril lluvioso, sacan a Mayo florido y hermoso (Castañón 1962 : 146) ; ou le léonais Xineiru nevosu, febreiru xelosu, marzu ventosu y'abril chuviosu sacan a mayo fluridu y'harmosu (Rúa 2014 : 164).

40 Gargallo (2012) l'utilise comme exemple de « paremiotipos concretos e representación territorial ». 
deuxième parémiotype, il énonce les résultats de la pluie féconde : Água de Fevereiro enche o celeiro ${ }^{41}$ (Ferreira 1999 : 214), en portugais ; A chuvia de febreiro fai o palleiro (Ferro 1995 : 206, num. 3158), en galicien; Agua de febrero, llena el granero (Martínez Kleiser 1995 : 431, num. 37.848), en espagnol ; Aigua en febrer, ordi en graner (DCVB : s. v. aigua, febrer), en catalan; ou Pioggia di febbraio, empie il granaio (Schwamenthal, Straniero 1991 : 408, num. 4450), en italien. Finalement, un bon résumé se trouve dans le proverbe catalan : Si plou el febrer, tot va bét2 (DCVB : s. v. febrer). En arrivant au mois de mars, il y a une coïncidence avec le proverbe minimal Março, encanar (Machado 1998 : 305), qui est peut-être en rapport avec la canalisation des eaux pour arroser.

Les cinq binômes qui suivent, d'avril à août, s'occupent d'un sujet central de la vie paysanne : la maturation et récolte des céréales. Effectivement, au mois d'avril, la croissance des céréales est évidente, d'où Abril, espigas mill3 (Marques 2004 : 48), qui peut complémenter le proverbe que nous commentons, Abril, espigar (Marques 2004 : 47). Aussi en galicien, formulé avec toute concision : Abril penduril ${ }^{44}$ (ALGa 2003: IV, 468, num. 436); ce proverbe minimal correspond à la première partie du dicton Abril, pendoril; maio, engraio; san Xoán, segaio (Vázquez 2003 : 121, num. 2856), qui, selon l'auteur, montre l'état du seigle dans les régions les plus chaudes de Galice : en avril l'épi pend, au mois de mai il est mûr, et en juin on peut faucher. Pendant le mois de mai, les épis vont mûrir, comme l'indique le proverbe minimal qui coïncide (sauf pour la virgule) avec le nôtre : Maio, engrandecer (Machado 1998 : 290). Un grand nombre de proverbes rattache le mois de juin à la moisson ; le portugais Junho, a ceifar (Machado 1998: 280) et les proverbes rattachables au parémiotype "Juin[,] faucille + poing " (v. Correas, Gargallo 2003 : 185-186), que nous nous bornerons à signaler en portugais, espagnol et catalan : Em Junho foice em punho (Machado 1998: 221) ; Junio, hoz en puño ; et Pel juny, la falç al puny (Parés 1999 : 126, num. 2487) (v. l'exemple 4). De son côté, juillet est le temps pour battre : Julho, debulhar (Machado 1998: 279); dans ce mois il se peut que la tâche du mois précédent (la moisson) continue : Nos dias de Julho, [/] Eu ceifo e debulho; [/] Se o vento vai dando, [/] Vou logo ensacando (Carrusca 1976 : III, 233 ; v. l'exemple 4). Le cycle des céréales se clôt en août: Agosto, engravelar (Machado 1998 : 67).

\footnotetext{
${ }^{41}$ Localisé à Trás-os-Montes et au Alto Douro.

42 En espagnol on arrive même à souhaiter : Venga febrero lluvioso, aunque salga furioso (Martínez Kleiser 1995 : 432, num. 37.851).

43 À comparer avec le quatrième composant de ce proverbe galicien : Xaneiro xiadeiro, febreiro quere corre-lo vougueiro, marzo espigarzo, en abril espigas mil, maio pardo, san Juan claro valen máis có boi i o carro (ALGa 2003 : IV, 462, num. 8 ; localisé à Xinzo de Limia, province d'Ourense).

${ }^{44}$ Localisé à Ortoño (province de A Coruña).
} 
Sans aucun doute, les travaux agricoles typiques de septembre sont les vendanges, comme l'indique la litanie analysée et aussi le proverbe minimal Setembro, vindimar (Machado 1998: 587). On en trouve de nombreux exemples dans le Calendario romance de refranes (v. Correas, Gargallo 2003 : 254); nous en citons seulement trois, en galicien, espagnol et occitan: Setembro[,] colle o viño e non durmas no camiño (Quintáns 1994 : 94) ; Septiembre el vendimiador, corta los racimos de dos en dos (Martínez Kleiser 1945 : 283) ; et $A u$ mes de setèmbre [,] [/] raisin soun bon à pèndre (Mistral 1979: II, 888, s. v. setèmbre).

(Exemple 7) Enero friolero, febrero ventolero, marzo airado, abril variado, mayo hermoso, junio fogoso, julio claro, agosto raro, septiembre extravagante, octubre abundante, noviembre llovedero y diciembre nevadero (Sánchez 1985 : 86, num. 1.324).

['Janvier frieux, février venteux, mars avec de l'air, avril colorié, mai joli, juin fougueux, juillet clair, août bizarre, septembre extravagant, octobre abondant, novembre pluvieux, décembre neveux']

À la différence des exemples 6 ou 9, Fontana (2019: 42) signalait le fait qu'aucun des douze proverbes minimaux qu'on pourrait extraire de l'exemple 7 (même pas *Enero friolero ou *Febrero ventolero, qui seraient renforcés par la rime) n'est attesté de manière isolée.

Enero el friolero entra soplándose los dedos (Martínez Kleiser 1995 : 469, num. 41.007) ['Janvier, frieux, entre en se soufflant les doigts']. Voici une ampliation du premier élément de notre proverbe, Enero friolero. 45 Suivent février et mars, tous deux caractérisés par les vents, ventolero et airado, respectivement (pensons au proverbe minimal Marzo, ventoso [Sánchez 1985 : 139, num. 1884]). Pour le binôme Abril variado (variado au sens 2 . du DLE'de varios colores' ; c-à-d. 'colorié'), il faudra rappeler le proverbe italien Il marzo tinge, april dipinge (Boggione, Massobrio 2007 : 17, num. I.3.4.1.21.), qui fait allusion à la renaissance de la nature, qui débute au mois de mars et arrive à sa splendeur en avril (v. l'exemple 11); dans ce sens, le proverbe minimal Aprile dipinge (Boggione, Massobrio 2007 : 20, num. I.3.5.1.8.a), nous renvoie à une filastrocca bien connue "Aprile, il gran pittore, va a spasso col pennello e mette già colore ». Un proverbe espagnol aussi bien connu augure qu'un mars venteux avec un avril pluvieux ont comme résultat un mai joli, l'élément suivant dans notre proverbe (mayo hermoso) : Marzo ventoso, y abril lluvioso,

\footnotetext{
45 Voyez, dans le commentaire de l'exemple 4, l'allusion à la «setmana dels barbuts », du 15 au 22 janvier, et qui est considérée la plus froide de l’an.
} 
hacen el mayo hermoso ${ }^{46}$ (Martínez Kleiser 1995 : 473, num. 41.389). L’adjectif 'fougueux' du mois de juin est sans doute en rapport avec la chaleur, tandis que le juillet 'clair' peut être une transposition de la caractéristique de juin au mois suivant (v. le parémiotype bien connu « Mayo pardo + junio claro $\rightarrow$ buen augurio »; v. l'Atles de ParemioRom). Aussi bien que les bizarreries et extravagances d'août et septembre sont difficiles à interpréter, l'abondance d'octobre peut s'expliquer par les greniers et celliers pleins avec le produit des récoltes et vendanges ; par contre, il n'y a pas de doute que novembre est un mois pluvieux.

Pour ce qui est du dernier binôme de la série, diciembre nevadero, il faut seulement penser à des proverbes comme en espagnol En diciembre, hielos y nieves (Martínez Kleiser 1995 : 473, num. 41.360) ou l'aranais Avens [/] posa dents ${ }^{47}$ ['Décembre [/] met les dents] (Gomis 1998 : 167). La neige de décembre, d'un autre côté, est un très bon présage pour la récolte de l'année suivante ; ainsi, Cuando en diciembre veas nevar, ensancha el granero y el pajar (Martínez Kleiser 1995 : 519, num. 45.480), en espagnol ; Si pel desembre neva bé, eixampla el graner i el paller (Amades 1951: 1002), en catalan [les deux avec le sens 's'il y a de la neige en décembre, tu peux agrandir ton grenier'] ; Dicembre nevoso, anno fruttuoso (Schwamenthal, Straniero 1991: 206, num. 2242), en italien; Neige de décembre [/] Est engrais pour la terre (Cellard, Dubois 1985 : 169), en français ; et Gerul şi neaua din decembrie vestesc grâu mult ['Le gel et la neige de décembre annoncent beaucoup de blé'] (Olteanu 2001 : 507), en roumain.

(Exemple 8) Gener ens portarà neus, febrer serà variable, pel març bufaran vents forts i l'abril serà agradable. Pel maig hi haurà floració [,] pel juny alguna tronada, al juliol farà calor i a l'agost forta secada. Setembre serà mig sec i l'octubre mig mullat, al novembre farà fred i al desembre vent gelat ${ }^{48}$ (Batllori et alii 1997 : 73).

\footnotetext{
46 Nous recueillons aussi quelques exemples galiciens, avec quelques traits non propres au galicien (p. ex. $i$ pour $e$, pour la conjonction copulative) ce qui peut être dû au fait que l'informant fait une traduction d'une parémie espagnole : Marzo ventoso i abril chuviñoso sacan a maio florido i hermoso (ALGa 2003 : IV, 465, num. 236; localisé à A Pobra do Brollón, province de Lugo), ou Marzo ventoso i abril chuvioso sacan a maio florido i hermoso (ALGa 2003 : IV, 466, num. 238 ; localisé à Meira, province de Lugo).

${ }^{47}$ C'est une partie prise du proverbe :
}

En auens que met dents;/en gè que s'í sè;/hereuè ven dam era pala en còtx e la 'n trè ['lla neige] En décembre, elle met dents; en janvier elle y prend siège ; /[mais] février vient avec une pelle au cou et l'enlève'] (Coromines $1990:$ 304-305, s. v. auens).

On notera, d'un autre côté, l'évolution sémantique 'Avent' > 'décembre, mois de l'Avent' de l'aranais, qu'on trouve aussi en haut aragonais (abiento [o aviento] EBA), basque (abendu OEHEI) et asturien (avientu DALIAEI).

${ }^{48}$ La source recueille cet exemple comme « embarbussament » ['virelangues'], ce qui ne semble pas exact. 
['Janvier nous portera neiges, février sera variable, en mars on aura vents forts et avril sera agréable. En mai, on aura les fleurs, en juin quelques tonnerres, en juillet on aura chaud et en août forte sécheresse. Septembre sera à moitié sec et octobre à moitié humide, en novembre il fera froid et en décembre vent gelé’]

Le premier élément de cette énumération catalane, Gener ens portarà neus, rappelle des proverbes catalans comme La neu al gener, s'hi asseu com un cavaller ['La neige en janvier; s'assied comme un chevalier'] (DCVB : s. v. neu) ou l'occitan aranais Je, [/] s'hi sé (v. note 47) ['Janvier, [/] [la neige] y prend siège'] (Gomis 1998 : 155). La neige, en début de l'an, est en général de très bon augure : La neu de gener / tot l'any va bé (Sanchis 1951 : 13, num. 28b) ; ou, plus concrètement, pour les récoltes : Neu de gener omple el graner (Amades 1951: 960), en catalan; Neve di gennaio, pieno il granaio (Schwamenthal, Straniero 1991: 329, num. 3584), en italien; ou, en français, avec une expression un peu diverse, Neige en janvier [/] Vaut fumier (Cellard, Dubois 1985 : 10). D’un autre côté, la variabilité de février est bien représentée dans plusieurs proverbes: de formulations minimales, comme l'espagnol Febrero, veletero $^{49}$ (Martínez Kleiser 1995 : 470, num. 41.041), ou le sarde Frearzu traitore (Spano 1997 : 159, s. v. Frearzu) ['Février, traître'], à proverbes plus élaborés, comme par exemple El febrer / nou cares té (Sanchis 1951 : 31, num. 10), en catalan ; En febreiro o louco, / ningún día se parece a outro ${ }^{50}$ (Rúa 2014 : 178), en galicien ; ou En febrero, el loco, ningún día se parece a otro (Martínez Kleiser 1995 : 470, num. 41.050), en espagnol. La transition entre un février variable et un mars venteux se trouve dans le proverbe asturien Febreru llocu y Marzu ventosu (Castañón 1962 : 164). Quatre proverbes minimaux expriment l'idée de mars venteux; un en occitan et trois en galicien :51 Mars, ventous (Mistral 1979 : II, 285, s. v. mars), Marzo airearzo52 (ALGa 2003 : IV, 465, num. 235), Marzo airoso ${ }^{53}$ (num. 234) et Marzo ventoso ${ }^{54}$ (num. 242). On trouve aussi des exemples plus longs et très parlants en portugais et en catalan : Dia de Março, dia de três ventos (Carrusca 1976 : III, 213) et Març, marçot, / ronca fins que és mort55 (Sanchis 1951: 51, num. 25). Cela mérite également de mentionner des proverbes comme l'asturien Cuando'n Marzu hay vientos

\footnotetext{
49 Veletero désigne quelque chose de variable comme une veleta ['girouette'] et ici il s'applique au mois de février, puisqu'il est censé être un mois inconstant.

50 Localisé à León.

51 Pour d'autres proverbes minimaux du mois de mars en galicien, v. Fontana (2017 : 53-54).

52 Localisé à Lubián (province de Zamora). La création ad hoc d'airearzo est intéressante.

53 Localisé à A Mezquita (province d'Ourense).

${ }^{54}$ Localisé à A Senra (province de Lugo) et à Rodeiro (province de Pontevedra).

55 Localisé à Cadaqués (province de Girona).
} 
juertes y Abril ye moyau y fríu, síguelos Mayu llozanu y floríu (Castañón 1962 : 82), qui renvoient au parémiotype bien connu « Marzo ventoso y abril lluvioso $\rightarrow$ mayo florido $»^{56}$ (v. l'Atles de ParemioRom).

L'abril, / mes gentil (Sanchis 1951 : 62, num. 1) ; effectivement, l'arrivée de ce mois annonce le début du bon temps : Abril finat, hivern passat (DCVB : s. v. hivern), en catalan; ou Abril empezado, invierno acabado (Pejenaute 1999: 30), en espagnol. En italien, de son côté, on dit Aprile, esce la vecchia dal cortile (Schwamenthal, Straniero 1991 : 43, num. 453), et, en galicien, on conseille En abril abre as portas e déixame ir (avec un sujet elliptique) (ALGa $2003:$ IV, 468, num. 419). Finalement, comme on dit en aranais, Entath mès d'abriu [/] tota bèstia que viu (CNLVA 1992 : 14). Pour ce qui est des fleurs de mai, on a déjà fait allusion au parémiotype «Marzo ventoso y abril lluvioso $\rightarrow$ mayo florido $", 57$ que parfois on limite à avril et mai : A abril con sus chaparrones, sigue mayo con sus flores (Martínez Kleiser 1995 : 469, num. 40.967).

Une fois arrivés à l'été, la litanie nous met en garde face aux tonnerres de juin, qui sont pourtant un bon présage pour la récolte : Trons pel juny, [/] any de fruits (Amades 1983 : III, 696). Pour juillet, la caractéristique est l'intense chaleur du soleil 58 comme le dit le proverbe El juliol, [/] l'amo del sol (Amades 1983 : IV, 354). En juillet, on a tellement chaud que Pel juliol, [/] amb poc foc bull el perol (Amades 1983: IV, 354); ou, en lombard, Luj, la terra la buj (Santoro 1998 : 46, num. 317), italianisé en Nel mese di luglio - la terra bolle (Hauser 1975 : 562). D'un autre côté, a l'agost forta secada nous rappelle le proverbe occitan Aost [/] Seca lo cosp ['Août [/] Sèche la vigne'] (Mistral 1979: I, 585, s. v. $\operatorname{cosp}$ ) ; pourtant, le temps sec en août est un bon présage, comme notent les proverbes espagnol, catalan et occitan : Dios te guarde / de polvo de mayo / y fango en agosto (Gomis 1998 : 204, note 65) ; El pagès només plora de dues coses : de l'eixut de maig i dels fangs d'agost (Sanchis 1951: 81, num. 37) et Diéu nous garde de la póussiero de mai e de la fango d'avoust! (Mistral 1979: II, 630, s. v. póussiero). En conclusion sur cet aspect, on a le proverbe romanche : D'in schetg Uost eis ei aunc mai vegniu paupra glieut, d'in bletsch bein ['D'un août sec n'en sont jamais venus des gens pauvres, d'un [août] mouillé, par contre, si'] (Hauser 1975 : 575).

\footnotetext{
56 Voici deux exemples de ce parémiotype, en galicien et espagnol respectivement: Marzo ventoso i abril chuviñoso sacan a maio florido i hermoso (ALGa 2003 : IV, 465, num. 236; localisé à A Pobra do Brollón, province de Lugo) et Marzo ventoso / y abril lluvioso / sacan a mayo florido y hermoso (Gomis 1998 : 195).

57 V. la note précédente et aussi l'Atles de ParemioRom.

58 Ce n'est pas par hasard qu'en roumain la désignation populaire du mois d'août est luna lui cuptor (DEX: s. v. cuptor), c'est à dire 'le mois du four'; Cuptor provient du latin CoctoriU ('étuve, four').
} 
Ensuite la formule Setembre serà mig sec nous rappelle que pendant ce mois il y a des épisodes extrêmes de pluie torrentielle et sécheresse, d'où le parémiotype connu «Septiembre, mes de comportamiento extremo : fuentes y puentes » (v. l'Atles de ParemioRom et Correas, Gargallo [2003: 252]), auquel correspondent les proverbes portugais, galicien et catalan suivants: Em Setembro ardem os montes e secam as fontes (Moreira 2003 : 108, num. 337), En setiembre arden os montes e secan as fontes ${ }^{59}$ (ALGa 2003 : IV, 471, num. 703, 705, 706) et El setembre s'enduu els ponts o eixuga les fonts (Amades 1951: 991). Pour ce qui est de l'octubre mig mullat, on notera que le nom populaire de ce mois en roumain est brumărel (DEX), dérivé de brumă 'gebre' ; l'humidité et la pluie apparaissent, comme on le voit dans le proverbe italien Ottobre è bello, ma tieni pronto l'ombrello (Schwamenthal, Straniero 1991 : 375, num. 4074).

Finalement, pour ce qui est du vent gelé de décembre, on n'a qu'à rappeler le proverbe français Le mois de l'Avent [/] Est sujet au vent (Cellard, Dubois 1985: 170), probablement d'origine occitane d'après la désignation «le mois de l'Avent » pour 'décembre'.

(Exemple 9) Gennaio zappatore, febbraio potatore, marzo amoroso, aprile carciofaio, maggio ciliegiaio, giugno fruttaio, luglio agrestaio, agosto pescaio, settembre ficaio, ottobre mostaio, novembre vinaio, dicembre favaio (Boggione, Massobrio 2007 : 10, num. I.3.1.8).

(Exemple 9a) Gennaro zappatore, febbraro potatore, marzo broccolaro, aprile cerasaro, maggio fiorellaro, giugno fruttarolo, lujo agrestaro, agosto persicaro, settembre ficaro, ottobere mostaro, novembere vinaro, decembere favaro (variante du Lazio) (Schwamenthal, Straniero 1991 : 259, num. 2814).

['Janvier laboureur, février élagueur, mars amoureux (ou riche en broccoli), avril riche en artichauts (ou cerises), mai riche en cerises (ou fleurs), juin riche en fruits, juillet riche en agresto, août riche en pêches, septembre riche en figues, octobre riche en moût, novembre riche en vin, décembre riche en fèves']

D’après Lapucci (2007 : 659, num. 404), qui recueille l'exemple 9,60 « Si indicano così i frutti principali o le faccende della campagna dei vari mesi dell'anno ». Ainsi, janvier est le mois pour labourer la terre pour les semences,

\footnotetext{
${ }^{59}$ Localisé à Fefiñáns (Cambados, province de Pontevedra) et à Oímbra (province d'Ourense).

${ }^{60}$ Lapucci introduit une césure après chaque mois et sa caractéristique. Le proverbe est aussi recueilli par Schwamenthal, Straniero (1991 : 259, num. 2814), avec des barres obliques au lieu de césure.
} 
comme le dit aussi le proverbe minimal Gennaio operaio (Boggione, Massobrio 2007 : 10, num. I.3.2.1.5.a.). Février est destiné à élaguer les vignobles ; la graphie ...febbraio potatore..., avec des points de suspension, que Lapucci (2007: 542, num. 494) présente comme proverbe minimal, prouve qu'il l'a tiré de notre litanie (exemple 9). D'un autre côté, l'adjectif amoroso pour le mois de mars rappelle le début de la saison de rut pour les oiseaux et autres animaux; carciofaio pour avril indique, bien entendu, la saison pour les artichauts.

Par la suite, les trois mois qui suivent apparaissent aussi dans les sources comme proverbes minimaux tronqués de la litanie ci-dessus : Maggio ciliegiaio (Boggione, Massobrio 2007 : 24, num. I.3.6.1.49 ; 80, num. II.6.2.1.4), en faisant allusion aux cerises ; ... giugno fruttaio...61 (Lapucci $2007: 683$, num. 786), pour la période des premiers fruits; et Luglio agrestaio ${ }^{62}$ (Boggione, Massobrio 2007 : 28, num. I.3.8.1.11 75, num. II.4.1.11), puisque « a luglio si vendemmia l'agresto (speciale tipo di vite, la cui uva si vendemmia prima della completa maturazione; se ne ricava un succo usato come bevanda e come condimento) ». Par contre, les pêches d'août et les figues de septembre n'existent pas sous forme de proverbe minimal (v. l'exemple 4, où les figues s'avancent au mois d'août).

Encore une fois, les trois derniers éléments sont attestés comme proverbes minimaux : Ottobre mostaio (Schwamenthal, Straniero 1991: 375, num. 4076 ; Lapucci 2007 : 1084, num. 704), c'est à dire le moût qui servira à remplir les tonneaux ; Novembre vinaio (Schwamenthal, Straniero 1991 : 354, num. 3856), faisant allusion au raisin tardif, qui fermente par séchage et duquel on obtient les vini passiti ; et Dicembre favaio ${ }^{63}$ (Schwamenthal, Straniero 1991 : 206, num. 2240), puisque c'est l'époque où on sème les fèves et, aussi, c'est l'époque où l'on utilise les fèves qu'on avait faites sécher pour l'hiver, par exemple pour la faverella, mets fait avec une pâte de farine de fève avec de l'eau et cuite au four.

On signalera que, pour ce qui est de la variante du Lazio (exemple 9a), il y a trois mois dont la caractérisation est différente que celle du proverbe italien standard (exemple 9) : mars, avril et mai ont les épithètes de broccolaro (c'est à dire, riche en broccoli), cerasaro (plein de cerises, affirmation qui est un peu surprenante parce qu'elle semble trop précoce) et fiorellaro (plein de fleurs).

\footnotetext{
61 À noter que, comme pour le cas précédent de ...febbraio potatore..., les points de suspension indiquent que le proverbe a été extrait de la litanie. V. aussi, la note 60 .

62 Lapucci (2007: 659, num. 404) note que «Gli aggettivi che qualificano i mesi sono tutti registrati nei dizionari, meno agrestaio, di cui ormai è sparito anche l'uso del corrispondente sostantivo agresto insieme alla cosa da questa indicata».

${ }^{63}$ La variante recueillie par Lapucci (2007 : 425, num. 324), ...dicembre favaio, avec les points de suspension, est tirée de la litanie parémique.
} 
(Exemple 10) Gennaro - pellicciaro, [/] febbraio - febbraieggia, [/] marzo - chi è pazzo vada scalzo, [/] aprile - non ti scoprire, [/] maggio vacci adagio, [/] giugno - non ti levare il cuticugno, [/] luglio - getta via $i$ panni e malanni, [/] agosto - piglia i panni che hai risposto, [/] settembre - solicino e gabbanino, [/] ottobre - chi ha panni si ricopre, [/] novembre - copriti sempre, [/] dicembre - sette bucce e sette cappucce (Lapucci 2007: 659, num. 407).

['Janvier - avec des pelisses, [/] février - il fait des choses typiques de février, [/] mars - le fou va pied-nu, [/] avril - ne pas se découvrir [/], mai - doucement, [/] juin - n'enlève pas le cuticugno, [/] juillet emporte les vêtements et maladies, [/] août - reprend les vêtements que tu as enlevés, [/] septembre - petit soleil et pardessus, [/] octobre qui a des vêtements qu'il se couvre, [/] novembre - couvre-toi tout le temps, [/] décembre - sept pelisses et sept capuchons']

L'exemple 10 est une litanie de douze conseils sur les vêtements (v. Gargallo 2007 ; on peut consulter aussi la banque de données ParemioRom en lançant la recherche "Ámbito temático general > Consejos de abrigo »), qui indiquent quels sont les vêtements qu'on doit utiliser pendant les douze mois de l'an. Ainsi, en janvier il faut mettre une pelisse pour combattre le froid. La création verbale ad hoc febbraieggiare (plus habituellement, febbreggiare) rend la variabilité de février; c'est la même formation qu'on trouve dans le proverbe minimal asturien Febrero, febreraes (Castañón 1962: 164; v. Fontana 2018 : 68-70). Pour les deux premiers mois, on peut suivre le conseil suivant: Gennaio e febbraio metti il tabarro (Lapucci 1995 : 207 ; v. l'exemple 4). Pour ce qui est de mars, la formule Di marzo [/] ogni matto vada scalzo (Lapucci 1995: 210), assez similaire à notre exemple, s'explique dans le proverbe suivant : Marzo, chi non ha scarpe vada scalzo, ma chi ce l'ha ne tenga di conto, perché l'inverno è sempre pronto (Schwamenthal, Straniero 1991: 295, num. 3237). Sinon, il faut se méfier de l'arrivée du printemps et des premiers jours chauds : Aprile non un filo, ${ }^{[64]}$ maggio va adagio65 (Schwamenthal, Straniero $1991: 44$, num. 459) ; il s'agit d'un conseil bien connu qui précise la date du 10 juin pour commencer à utiliser des vêtements plus légers : Fins a setanta d'abril no et llevis un fil (Parés 1999: 111, num. 1869) et Hasta el cuarenta de mayo, no te quites el sayo, y si junio es ruín, hasta el fin (Martínez

\footnotetext{
64 Il s'agit sans doute d'une adaptation parémiographique à l'italien des proverbes des dialectes septentrionaux où les issues d'APRILE i FILU riment à cause de la perte des voyelles finales. Voyez, dans l'Atles de ParemioRom, le parémiotype « Abril en rima con el tipo léxico heredero de FILU ».

65 Il faut rappeler le proverbe galicien Garda o teu saio para maio (Gargallo 2007 : 99). Aussi en italien, avec la création ad hoc maggione: Né di maggio né di maggione, non ti levare il pelliccione (Schwamenthal, Straniero 1991 : 321, num. 3497).
} 
Kleiser 1945 : 259), en catalan et espagnol, respectivement. Justement ce dernier conseil du proverbe espagnol de garder des vêtements chauds pendant tout le mois de juin s'accorde avec le conseil de notre litanie sur le cuticugno, ${ }^{66} c^{\prime}$ est à dire, « un giubbetto o una sopravveste che portavano gli uomini in casa o per lavori al chiuso » (Lapucci 2007 : 659, num. 406).

Après la première moitié de l'année où il faut garder des vêtements chauds, le mois de juillet marque un tournant où on peut laisser en arrière les vêtements plus lourds et les maladies de l'hiver. Cette pause est pourtant bien courte, puisque, comme on dit en portugais, Primeiro de Agosto, primeiro de inverno (Carrusca 1976 : III, 234 ; v. la note 33) ; et, ainsi, au mois d'août il faut récupérer les vêtements qu'on avait abandonnés en juillet. $\mathrm{Si}$, en septembre, on doit utiliser le pardessus, en octobre il est conseillé de mettre des vêtements épais et en novembre on utilise tous les vêtements d'hiver puisque, comme l'indique le proverbe italien, Per i Santi [/] manicotto e guanti (Antoni, Lapucci 1993: 249); en décembre il faut endosser tous les vêtements que nous possédons ('sept pièces et sept capuchons'). En résumé, des conseils plutôt conservateurs étant donné que, en somme, on ne devrait être sans abri qu'au mois de juillet. Comme complément, plus facile à rappeler, on a le conseil du proverbe italien suivant: Quando l'albero si spoglia véstiti, quando l'albero si veste spogliati (Schwamenthal, Straniero 1991: 430, num. 4709) ['Quand l'arbre est habillé, déshabille-toi ; quand l'arbre est déshabillé, habille-toi'].

(Exemple 11) Gennaio mette ai monte la parrucca, [/] febbraio grandi e piccoli imbacucca, [/] marzo libera il sol di prigionia, [/] april di bei colori orna la via. [sic] [/] maggio vive tra musiche d'uccelli, [/] giugno ama i frutti appesi ai ramoscelli, [/] luglio falcia le messi al solleone, [/] agosto, avaro, ansando le ripone, [/] settembre dolci grappoli arrubina, [/] ottobre di vendemmia empie la tina, [/] novembre ammucchia foglie per la terra, [/] dicembre ammazza l'anno e lo sotterra (Lapucci 2007 : 659-660, num. 408).

Nous ne nous attarderons pas sur ce proverbe puisque Lapucci (2007: 660, num 408) croit qu'il « Pare, più che una forma proverbiale (tranne forse il primo verso), una poesiola di fattura dotta, nata per uso scolastico». Effectivement, plutôt que des prédictions, travaux agricoles ou des conseils sur les vêtements, cette litanie nous présente des lieux communs : la renaissance de la nature au printemps (avril-mai), les vendanges et l'élaboration du vin (septembre-octobre) ou, topique poétique, la chute des feuilles en automne

66 Nous prenons le proverbe Di giugno [/] levati il cuticugno [/] e se non ti pare [/] tornalo a infilare d'Antoni, Lapucci (1993 : 172). 
(novembre). Il faut pourtant mettre en relief, comme le fait déjà Lapucci, le premier vers, Gennaio mette ai monte la parrucca, qui fait allusion aux sommets des montagnes cachés par le brouillard ou les nuages, image qui a suscité la métaphore de la montagne avec perruche (l'oiseau), bonnet, cape, capuchon, etc, qui est un des parémiotypes les plus connus dans la Romania. ${ }^{67}$

\section{Conclusions}

L'observation du cycle de l'an résulte en une tradition italienne assez particulière ; l'éminent parémiologue italien Carlo Lapucci et Anna Maria Antoni (1993) expliquent cette tradition qui reçoit des noms comme Il contrasto dei mesi (1993 : 11-34), et qu'ils présentent comme suit :

La corona dei mesi, detta anche la mascherata, il contrasto dei mesi, mascherata del calendario, è un'antichissima composizione che ripete sul piano della poesia quello che sono le serie di dodici figure, ciascuna rappresentante un mese dell'anno, nella pittura, nella scultura, nel bassorilievo, nelle formelle, nelle xilografie e nelle incisioni (Antoni, Lapucci 1993 : 11).

Une de ces manifestations consiste en une pièce de théâtre populaire où le "père », l'an, présente ses douze "fils", les mois, avec deux quatrains comme introduction et, après, les traits qui les caractérisent :

Io sono il padre di dodici figli

e tutti quanti sono mortali;

vesto di rose, di fronde e di gigli,

io sono il padre di dodici figli,

Non c'è n'è uno all'altro uguale:

scema la notte e sale il di,

poi scema il dì e la notte sale:

il mondo è sempre fatto così (...)

Chaque mois est caractérisé par une formule débutant par «Io son(o) + nom du mois » :

Io son Gennaro nel canto del foco;

Io son Febbraro che porto il sereno;

${ }^{67}$ Pour des détails on peut consulter l'Atles de ParemioRom ( « Montañas con capa $\rightarrow$ Consejos de abrigo ») et les 266 proverbes correspondant à ce parémiotype de la banque de données. 
Io sono Marzo che vengo col vento;

Io sono Aprile ridente e gentile;

Io sono Maggio, di tutti il più bello;

Io sono Giugno che mieto lo grano;

Io sono Luglio che aspetta il villano;

Io sono Agosto e so' il più galante;

Io son Settembre, il mese cortese;

Io sono Ottobre che faccio il vino;

Io son Novembre che porta la bruma;

Io son Dicembre che Dio vien dal cielo.

Et clôt encore le père des douze fils :

Io sono il padre di dodici figli

e tutti quanti sono mortali;

vesto di rose, di fronde e di gigli,

io sono il padre di dodici figli.

La série de dictons avec tous douze mois de l'an que nous avons présentée rappelle cette tradition italienne du contrasto dei mesi. Dans les caractérisations que nous avons recueillies il y a souvent des formulations bimembres, mais aussi d'autres formulations plus longues; l'économie des compositions individuelles est essentielle pour arriver à bâtir ces longues litanies pour les douze mois.

D'un autre côté, il est bien connu que des proverbes minimaux ou bimembres s'intègrent dans ces constructions parémiques majeures. Les litanies des douze mois sont le résultat des traditions parémiographiques, plutôt que de la tradition orale où elles semblent insolites. Elles sont, en outre, en contraste avec les proverbes minimaux (v. Fontana 2019).

\section{BIBLIOGRAPHIE}

AIS = Jaberg, Karl, Jud, Jakob, 1928-1940, Sprach- und Sachatlas Italiens und der Südschweiz, 8 vol., Zofingen, Ringier. Consultable en ligne : <https://www3.pd.istc.cnr.it/navigais-web/>. Alcalá Venceslada, Antonio, 1980, Vocabulario andaluz, Madrid, Editorial Gredos. ALeCMan = García Mouton, Pilar, Moreno Fernández, Francisco (dirs.), 2003, Atlas Lingüístico (y etnográfico) de Castilla - La Mancha, Universidad de Alcalá. Consultable en ligne : <http://www2.uah.es/alecman>. 
$A L G a=$ García, Constantino, Santamarina, Antón (dirs.), 2003, Atlas Lingüístico Galego, vol. IV (Léxico. Tempo atmosférico e cronolóxico), A Coruña, Fundación Pedro Barrié de la Maza.

Amades, Joan, 1951, Folklore de Catalunya, vol. II (Cançoner. Cançons - refranys endevinalles), Barcelona, Editorial Selecta.

Amades, Joan, 1982-1983 [1950-1956], Costumari català. El curs de l'any, 2a. edició en facsímil, Barcelona, Salvat Edicions (5 vol.).

Antoni, Anna Maria, Lapucci, Carlo, 1993, 30 dì conta novembre... I proverbi dei mesi, Milano, Garzanti Editore s.p.a.

Armanac Gascon 1985, 1984, Pau, Institut Culturau de Gasconha \& Société Nouvelle d'Éditions Régionales et de Diffusion.

Batllori, Josep, Batllori, Jordi, Ruiz, Àngels, 1997, Què t'empatolles? Recull d'embarbussaments, Barcelona, Editorial La Galera.

Bellonzi, Fortunato, 2000 [1995], Proverbi toscani, Firenze, Giunti Editore.

Boggione, Valter, Massobrio, Lorenzo, 2007, Dizionario dei proverbi, Torino, UTET.

Bonafè i Barceló, Francesc, 2009, Paremiologia del calendari (Costums, llegendes, cançons, proverbis, glos[s]es i refranys], edició a cura de Guillem Villalonga, Palma, Edicions Documenta Balear.

Botezatu, Grigore, Hâncu, Andrei, 2003, Dicţionar de proverbe şi zicători românești, ediţia a III-a, București-Chișinău, Editura Litera internaţional.

Carrusca, Maria de Sousa (coord.), 1974-1976, Vozes da sabedoria, Lisboa, Edição da coordenadora (3 vol.).

Cassano, Joseph, 1988 [1914], La vie rustique et la philosophie dans les proverbes et dictons valdôtains, 3ème. édition, Turin-Aosta, F. Casanova, J. Brivio \& J. Vittaz.

Castañón, Luciano, 1962, Refranero asturiano, Oviedo, Diputación de Oviedo/Instituto de Estudios Asturianos (CSIC).

Cellard, Jacques, Dubois, Gilbert, 1985, Dictons de la pluie et du beau temps, Paris, Éditions Belin.

CNLVA = Centre de Normalisacion Lingüistica dera Val d'Aran, 1992, Arrepervèris, Lleida, Pagès Editors.

CORDE = Real Academia Española, Corpus diacrónico del español. Consultable en ligne : $<$ http://corpus.rae.es/cordenet.html>.

Coromines, Joan, 1990, El parlar de la Vall d'Aran. Gramàtica, diccionari i estudis lexicals sobre el gascó, Barcelona, Curial Edicions Catalanes.

Correas, Gonzalo, 2000 [1627], Vocabulario de refranes y frases proverbiales, Edición de Luis Combet. Revisada por Robert James y Maïté Mir-Andreu, Madrid, Editorial Castalia.

Correas Martínez, Miguel, Gargallo Gil, José Enrique, 2003, Calendario romance de refranes, Barcelona, Edicions de la Universitat de Barcelona.

DALIAEl = DALIA = Academia de la Llingua Asturiana, 2000, Diccionariu de la llingua asturiana, Oviedo, Academia de la Llingua Asturiana. Consultable en ligne: $<\mathrm{http}$ ///www.academiadelallingua.com/diccionariu>.

DCECH = Corominas, Joan, Pascual, José Antonio, 1980-1991, Diccionario crítico etimológico castellano e hispánico, Madrid, Editorial Gredos (6 vol.). 
DCVB = Alcover, Antoni M., Moll, Francesc de B., 1930-1962, Diccionari català-valenciàbalear, Palma de Mallorca, Moll (10 vol.). Consultable en ligne : <http://dcvb.iecat.net/>.

Del Fabro, Adriano, 2000, Proverbi e modi di dire del Friuli, Colognola ai Colli, Libri Demetra.

$D E X$ = Academia Română \& Institutul de Lingvistică « Iorgu Iordan », 1998, Dicționarul explicativ al limbii române. Consultable en ligne : <https://dexonline.ro/>.

$D L E=$ Real Academia Española, Diccionario de la lengua española. . Consultable en ligne : <https://dle.rae.es/>.

Doval, Gregorio, 1997, Refranero temático español, Madrid, Alba Libros.

$E B A=$ Endize de bocables de l'aragonés seguntes os repertorios lesicos de lugars y redoladas de l'Alto Aragón, 1999, Uesca, Instituto de Estudios Altoaragoneses (4 vol.).

Farnés, Sebastià, 1992-1999, Paremiologia catalana comparada, Edició a cura de Jaume Vidal Alcover, Magí Sunyer i Josep Lluís Savall amb la col·laboració de Josep M. Pujol, Barcelona, Columna Edicions (8 vol.).

Ferreira, Joaquim Alves, 1999, Miscelânia, vol. IV (Literatura Popular de Trás-osMontes e Alto Douro) Vila Real, Minerva Transmontana Tipografia, Lda.

Ferro Ruibal, Xesús, 1995, Refraneiro galego básico, Vigo, Editorial Galaxia.

Fontana, Joan, 2017, «Marzo, iguarzo. Achega aos refráns mínimos galegos », in Cadernos de Fraseoloxía Galega 19, p. 47-70. Consultable en ligne: <http://www.cirp.gal/w3/publicacions/pub-0490.html>.

Fontana, Joan, 2018, "Quen guarda, faya. Aproximación a los refranes mínimos asturianos », in Archivum, 58, p. 61-91. Consultable en ligne: <https://www.unioviedo.es/reunido/index.php/RFF/article/view/12710/pdf_1>.

Fontana, Joan, 2019, "Mundo inmundo. Aproximación a los refranes mínimos en español », in Paremia, 28, p. 37-47. Consultable en ligne : <https://cvc.cervantes.es/lengua/paremia/pdf/028/004_fontana.pdf>.

Fraguas, Antonio, 2006, Polas catro témporas. Colaboracións en La Voz de Galicia e 0 Correo Galego (1992-1999), Santiago de Compostela, Consello da Cultura Galega.

Gargallo, José Enrique, 1999, « Doce días para doce meses. De meteorología popular en la Romania », in Revista de Dialectología y Tradiciones Populares, 54 (2), p. 231-267.

Gargallo, José Enrique, 2007, «Garda o teu saio para maio. Consellos de abrigo no calendario romance de refráns », in Cadernos de Fraseoloxía Galega, 9, p. 95-112. Consultable en ligne : <http://www.cirp.gal/pub/docs/cfg/cfg09_05.pdf>.

Gargallo, José Enrique, 2009, "Octubre vinatero, padre del buen enero. Personificación de los meses en el calendario romance de refranes », Teddy Arnavielle, Christian Camps (éds.), Discours et savoirs sur les langues dans l'aire méditerranéenne, Paris, Éditions L'Harmattan, p. 303-319.

Gargallo, José Enrique, 2012, «Do ALGa a BADARE. Refráns do calendario e meteorolóxicos, xeoparemioloxía galega e romance ", in Novas achegas ao estudo da cultura galega, II, A Coruña, Universidade da Coruña, p. 411-420.

Gargallo, José Enrique, 2018, "Mai e rai. Refranes romances del mes de mayo », in Fonetică și dialectologie, 37, p. 29-47. Consultable en ligne : <https://www.lingv.ro/images/FONETICA-2018-TIPO.pdf>.

Gippini Escoda, Enrique, 1991, Refraneiro galego (Escolma), Sada (A Coruña), Ediciós do Castro. 
Gomis i Mestre, Cels, 1998, Meteorologia $i$ agricultura populars. Recull d'aforismes, modismes, creences $i$ supersticions referents a la meteorologia $i$ a l'agricultura a l'entorn dels anys 1864 a 1915 [Edició notablement augmentada amb gran nombre de confrontacions a cura de Cels Gomis i Serdañons], Barcelona, Alta Fulla Editorial.

Hauser, Albert, 1975, Bauernregeln. Eine schweizerische Sammlung mit Erläuterungen von Albert Hauser, Zürich-München, Artemis Verlag.

Lapucci, Carlo, 1995, Cielo a pecorelle. I segni del tempo nella meteorologia popolare, Cernusco, Casa Editrice Garzanti.

Lapucci, Carlo, 2007, Dizionario dei proverbi italiani, Milano, Mondadori Editore.

Leite de Vasconcelos, José, 1994-2007, Etnografia Portuguesa, Lisboa, Imprensa Nacional Casa da Moeda (10 vol.).

Machado, José Pedro, 1998, 0 grande livro dos provérbios, 2. ${ }^{\text {a }}$ edição, Alfragide, Casa das Letras.

Marques da Costa, José Ricardo, 2004, O livro dos provérbios portugueses, 2. a edição revista e ampliada, Lisboa, Editorial Presença.

Martínez Kleiser, Luis, 1945, El tiempo y los espacios de tiempo en los refranes, Madrid, Librería General de Victoriano Suárez.

Martínez Kleiser, Luis, 1995 [1953], Refranero general ideológico español, Madrid, Editorial Hernando.

Martinis, Mario, 2010, Proverbi del Friuli, Udine, Editoriale FVG spa.

Mistral, Frédéric, 1979 [1878-1886], Lou Tresor dóu Felibrige ou Dictionnaire ProvençalFrançais, Édition du centenaire sous la direction de V. Tuby, Genève - Paris, Slatkine \& Édition de l'Unicorne (2 vol.).

Montreynaud, Florence, Pierron, Agnès, Suzzoni, François, 1993, Dictionnaire de proverbes et dictons, Paris, Éditions Le Robert.

Moreira, António, 2003, Provérbios portugueses, 5.a edição, Lisboa, Editorial Notícias.

OEHEl = Mitxelena, Koldo, 2019, Orotariko Euskal Hiztegia/Diccionario general vasco, Bilbao, Publicaciones de Euskaltzaindia. Consultable en ligne: <https://www.euskaltzaindia.eus/index.php?option=com_oehberria\&task=bilak eta\&Itemid=413\&lang=eu>.

Olteanu, Antoaneta, 2001, Calendarele poporului român, București, Editura Paideia.

Ostermann, Valentino, 1995, Proverbi friulani raccolti dalla viva voce del popolo, Vago di Lavagno, Del Bianco Editore.

Palacios Rasal, Liena, Baos Muñoz, Chan, 2010, Bocabulario de Murillo de Galligo, Uesca, Publicazions d'o Consello d'a Fabla Aragonesa.

ParemioRom = Paremiología romance refranes meteorológicos y territorio. Consultable en ligne : <http://stel.ub.edu/paremio-rom/es>.

Parés, Anna, 1999, Tots els refranys catalans, 2a edició, Barcelona, Edicions 62.

$P C C D=$ Pàmies i Riudor, Víctor, 2020, Paremiologia catalana comparada digital . Consultable en ligne: <https://pccd.dites.cat/>.

Pejenaute Goñi, Javier María, 1999, Los refranes del Tiempo de Navarra, Pamplona, Caja de Ahorros de Navarra.

Quintáns Suárez, Manuel, 1994, Anuario de tradicións galegas, Santiago, Editorial Tambre \& Xunta de Galicia. 
Reis, José Alves, 1995, Provérbios e Ditos Populares, Lisboa - Porto, Litexa Editora.

Rodríguez Marín, Francisco, 1896, Los refranes del almanaque. Recogidos, explicados y concordados con los de varios paises [sic] románicos, Sevilla, Imp. de Francisco de P. Díaz.

Rúa Aller, F. Javier, 2014, Refranes del tiempo en León, León, Eolas Ediciones.

Rusu-Păsărin, Gabriela, 2006, Calendar popular românesc, Craiova, Editura Scrisul Românesc.

Sabaté i Rodié, Teresa, Culla i Perarnau, Rita, 1994, Els mesos de l'any, 3a edició, Barcelona, Editorial Salvatella.

Sampieri, Martinu, 1996, Parlà di u Sartinesu, [s. l.] [s. é.].

Sánchez Egea, José, 1985, El libro de los refranes de la temperie, Madrid, Instituto Nacional de Meteorología.

Sanchis Guarner, Manuel, 1951, Calendari de refranys, Barcelona, Editorial Barcino.

Santoro, Caterina, 1998, Proverbi milanesi, Firenze, Giunti Editore.

Sbarbi, José María, 1980 [1891], Monografía sobre los refranes, adagios y proverbios castellanos y las obras ó fragmentos que expresamente tratan de ellos en nuestra lengua, Madrid, Ediciones Atlas.

Schwamenthal, Riccardo, Straniero, Michele L., 1991, Dizionario dei proverbi italiani, Milano, Rizzoli Libri.

Spallicci, Aldo, 1996, Proverbi romagnoli, Firenze, Giunti Editore.

Spano, Giovanni, 1997 [1871], Proverbi sardi trasportati in lingua italiana e confrontati con quelli degli antichi popoli, a cura di Giulio Angioni, Nuoro, Ilisso Edizioni.

Tagliavini, Carlo, 1963, Storia di parole pagane e cristiane attraverso i tempi, Brescia, Morcelliana Editrice.

Treccani $=$ Istituto della Enciclopedia Italiana, Vocabolario. Consultable en ligne : $<$ http://www.treccani.it/vocabolario/> .

Vázquez Saco, Francisco, 2003, Refraneiro galego e outros materiais de tradición oral, in Cadernos de Fraseoloxía Galega, 5, Santiago de Compostela, Xunta de Galicia, Consellería de Educación e Ordenación Universitaria \& Centro Ramón Piñeiro para a Investigación en Humanidades.

Zamora Mosquera, Federico, 1972, Refráns e ditos populares galegos, Vigo, Editorial Galaxia.

Zanne, Iuliu A., 2003-2004 [1895-1903], Proverbele românilor din România, Basarabia, Bucovina, Ungaria, Istria și Macedonia, București, Editura Librăriei Socecu \& Comp (10 vol.). 